\title{
A NOVEL APPROACH FOR FAULTY NODE DETECTION WITH THE AID OF FUZZY THEORY AND MAJORITY VOTING IN WIRELESS SENSOR NETWORKS
}

\author{
Saeed Javanmardi ${ }^{1}$, Ali Barati ${ }^{2}$, Seyyed Jalaleddin Dastgheib ${ }^{3}$, \\ Iman Attarzadeh ${ }^{4}$ \\ 1,2,3,4 Department of Computer Engineering, Dezful branch, Islamic Azad University, \\ Dezful, Iran \\ Saeedjavanmardi@gmail.com \\ abaratieiaud.ac.ir \\ dastqeibegmail.com \\ attarzadeh@iaud.ac.ir
}

\begin{abstract}
Wireless sensor networks (WSN) consist of many nodes that are usually created to identify environmental incidents. Each of these nodes includes sensor, processor, communication components (antenna), small memory, and a source of energy. In wireless sensor networks' applications, faulty nodes always cause crucial problems and error in the network. For example, failure of some nodes may pull some parts of network into isolation, or in a worse case, the entire network may stop working, or decision about the occurrence of events may be corrupted. This paper proposes a new method to detect faulty nodes in the WSN using fuzzy logic and majority voting technique. By using the attributes of the fuzzy logic like interpretation, etc. in corporate majority voting technique can overcome the problem of faulty nodes, efficiently. In the proposed method, the fuzzy logic uses to identify the ratio of the faulty nodes in the network and in each sub-network. Using the calculated ratio and an effective decision making system such as majority voting is used to detect the faulty node in WSN. Using this effective method increases the percentage of detecting faulty nodes, which resulted in decreasing computational complexity, end to end delay and energy consumption in WSN.
\end{abstract}

\section{KEYWORDS}

Wireless sensor networks, faulty node, fuzzy theory, majority voting

\section{INTRODUCTION}

Wireless sensor networks (WSN) consist of many sensor nodes that sense its perimeter amount. A major usage of WSN is to observe and report the incidents of events of interest such as environment temperature, etc. [1][2][3][4]. In different WSN applications, [5] nodes because of physical causes or hardware crash may always have failure, which causes some problems in network functionality thus detecting failure nodes is really important. Because of the behaviour of WSN, the environment should keep out the faulty sensors to guarantee the network QOS [6]. This paper proposes a majority voting system based on fuzzy logic with low energy consumption. In the proposed method if a significant difference between the number of sensed " 1 " of a node and 
the number of sensed " 1 " of its neighbours at a specified interval, then this node is identified as a faulty node.

Three types of sensor faults are usually considered in the existing fault detection methods that sense 0/1 decision predicates. In fault detection type one, a faulty sensor node is frozen to sense a fixed local decision ' 0 ' regardless of the real observation which is named stuck-at-zero fault. The second type of fault detection method is stuck-at-one fault that a faulty node always senses a fixed decision ' 1 '. In the third type of fault detection method, a faulty sensor node senses " 0 " or " 1 " randomly, which does not reflect the reality and it is called random fault [7][8]. Fuzzy logic can be used to detect faulty nodes. In WSNs, fuzzy logic has been used to improve decisionmaking, reduce resource consumption, and increase performance. Some of the areas it has been applied to are cluster-head election [9, 10], security [11, 12], data aggregation [13], routing [14, 15], MAC protocols [16], and QoS [17, 18]. An overview of fuzzy logic will be done in Section 3 . We in this paper propose an efficient method by using fuzzy logic to detect faulty nodes.

The remaining part of this paper is organized as follows. Section 2 discusses the majority voting and fuzzy theory as background. The proposed faulty node detection method is presented in section 3. The comparison of the proposed method with the other fault detection methods is discusses in section 4. Finally the conclusion and the future work are discussed in section 5.

\section{BACKGROUND}

\subsection{MAJORITY RULE}

Majority voting is a technique to combine decisions of several classifiers or decision makers to improve the recognition process [19], [20]. The fundamental opinion in voting is to get a minimum number of nodes to consent to an operation before obligation [21].

According to majority rule, a node maintains its own measurement only when this result is the majority result within its neighbourhood [22]. It means if a sensor's treatment is different from the treatment of the majority of the inclosing sensors, it is a faulty sensor. A table for registering the neighbour nodes behaviour is considered. For example if a node is sensed " 1 " only once in a ten times sensed period rate would be 1/10. This issue is shown in Figure 1.

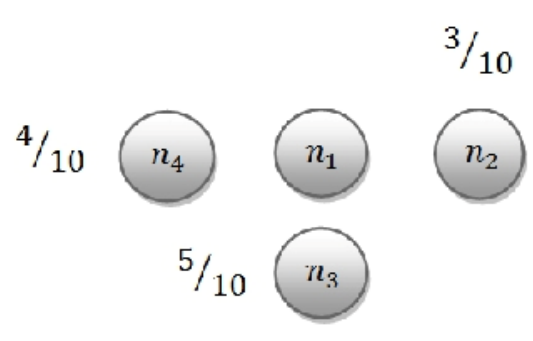

Fig 1: Assigning the rate based on the sensed "1"

Figure 1 describes the sensed value of sensors in a specific period. If the sensed value of the specific sensor has a significant difference, its neighbour nodes would be detected as faulty node. Figure 2 shows how the neighbour nodes transmit the number of sensed "1" to $n_{1}$ (the specific node). 


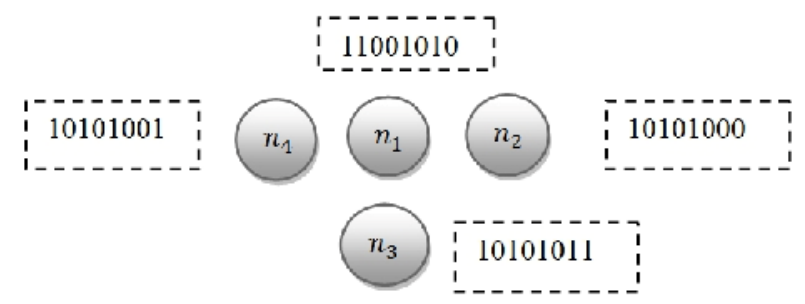

Fig 2: The number of sensed " 1 " in a specific period of time

\subsection{Fuzzy logic}

Fuzzy logic is based on probabilistic logic $[26,27]$. This logic is related to the precept of not exact reasoning, with exact reasoning. The part of a fuzzy rule before THEN is called predicate or antecedent, while the part following THEN is referred to as consequent. The combined truth of the predicate is determined by implication rules such as MIN-MAX and bounded arithmetic sums That is, it finds a single crisp output value from the solution fuzzy space. Common defuzzification techniques are centroid, composite maximum, composite mass, etc.

\section{PROPOSED METHOD}

As it stated above, the majority of sensed value can determine if the specific node is a faulty node or not. Figure 3 shows the structure of proposed fault detection method using fuzzy logic.

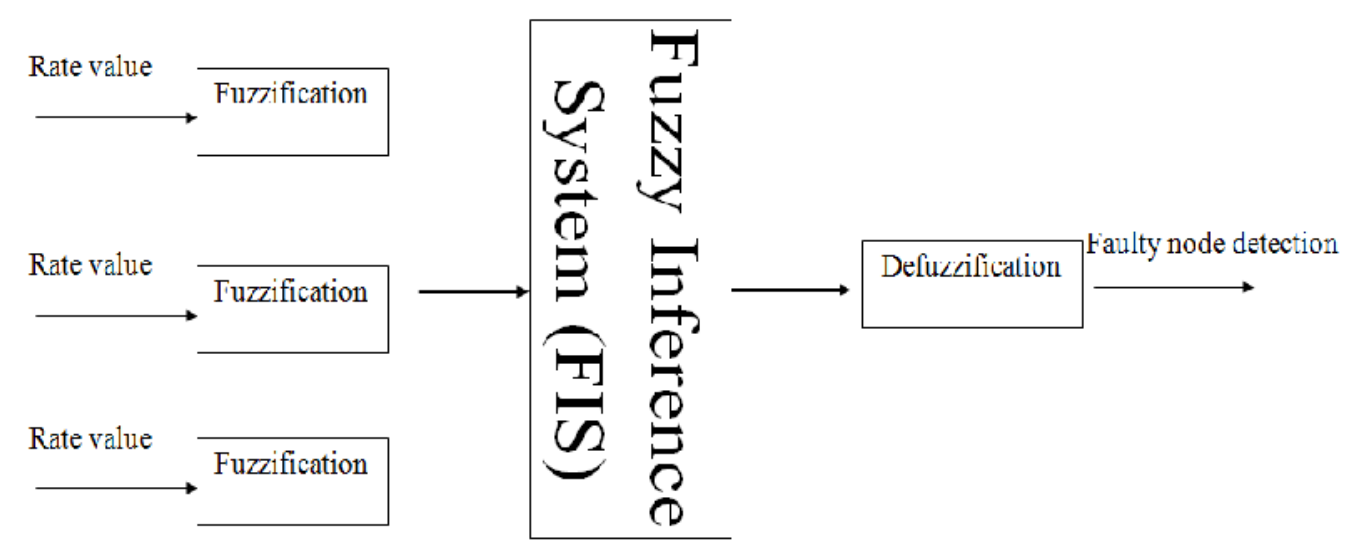

Fig 3: Fuzzy inference system that is used in our scheme

In the proposed fault detection method, the number of sensed " 1 " by neighbour nodes will be transmitted to specific node. One of the good advantages of this method is that as the rate of sensed "1" by neighbour nodes (Low, Medium, and High) will be transmitted, instead of transmitting all sensed data, energy consumption is reduced, efficiently. The amount of sensed data is in three levels, so 2 bits can transmit it. It means $2^{2}$ states for transmitting " 00 ", "01", "10", and "11", as shown in Figure 4. 


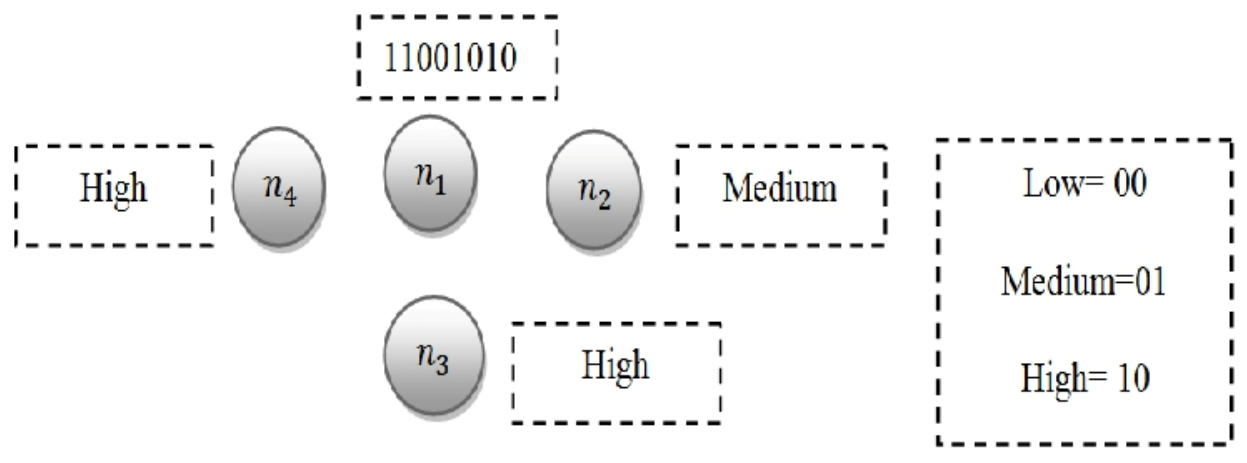

Fig 4: Reducing the number of transmitting by neighbor nodes

In the proposed model, the key point is the rate of a neighbour node, which based on the fuzzy logic converts to multi levels. For example, if the rate of neighbour node were 6, it would be placed in low level, medium level, and high level with different membership degrees. The membership function and membership degrees are uses to determine each neighbour in which level would be placed. A membership function is a curve that shows how a point in input space will be mapped to the membership value in output space. $\lambda$ shows the membership degree and it is a number between 0 and 1 . For example, 3 with the membership degree of 0.4 belongs to medium level; with the membership degree of 0.6 belongs to low level; and with the membership degree of 0 belongs to high level, as shown in equation Eq. (1).

$\mu_{A} \operatorname{High}(X)=0.0, \mu_{A} \operatorname{Medium}(X)=0.4, \mu_{A} \operatorname{Low}(X)=0.6$

$\mu_{A}(\mathrm{x})=\operatorname{Degree}(\mathrm{x})$ in $\mathrm{A} \quad \forall \mathrm{x} \in \mathrm{X}:{ }_{\mathrm{A}}(\mathrm{x}): \mathrm{X} \rightarrow[0,1]$

Obviously, the levels with the highest membership degree will be selected as the sensed level of neighbour node. Figure 5 shows the determination of membership degree in the proposed method.

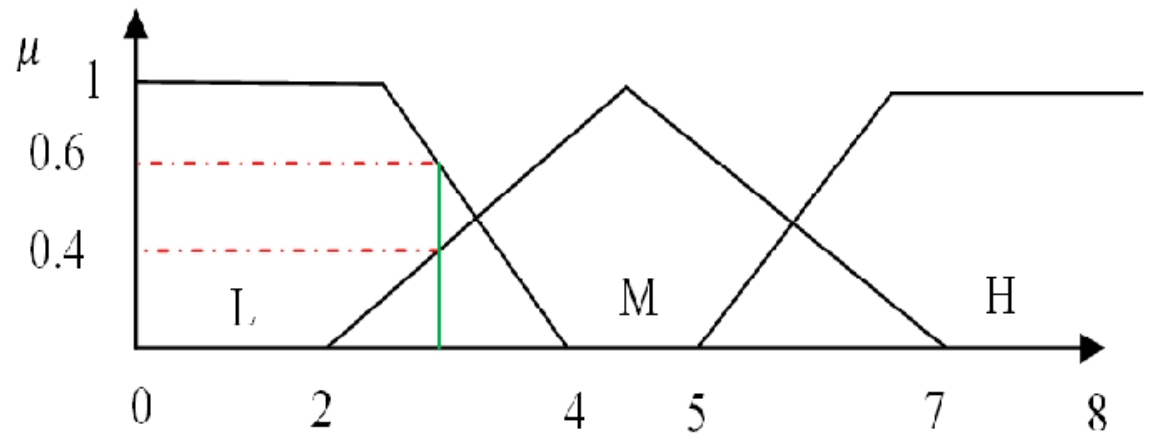

Fig 5: Membership function of neighbor node in fuzzy levels

Fuzzification process takes the crisp inputs from each of input variables and determines the degree to appropriate fuzzy sets. Table 1, shows samples of fuzzy inference rules, which used in the fuzzification process. Also, the Mamdani technique is used in the fuzzification process [28]. 
International Journal Of Advanced Smart Sensor Network Systems ( IJASSN ), Vol 2, No.4, October 2012

Table 1: Samples of fuzzy rules

\begin{tabular}{|l|l|l|l|l|}
\hline$n_{1}$ & $n_{2}$ & $n_{3}$ & $n_{4}$ & Result \\
\hline High & Low & Low & Low & Very High faulty \\
\hline High & Low & Medium & Low & High faulty \\
\hline High & Low & High & Low & Medium faulty \\
\hline Medium & Low & Low & Low & High faulty \\
\hline Medium & Low & Medium & Low & Low faulty \\
\hline Medium & Medium & High & Medium & Low faulty \\
\hline Low & Low & Low & Low & Very Low faulty \\
\hline Low & Low & High & Low & Low faulty \\
\hline Low & High & Medium & High & Very High faulty \\
\hline
\end{tabular}

Fuzzy inference is a process that maps input variables into output variables using fuzzy logic. Each fuzzy rule is weighted between " 0 " and " 1 " then using Mamdani fuzzy inference system and T-Norm operator, the output fuzzy set is generated.

A t-norm is a function $\mathrm{T}:[0,1] \times[0,1] \rightarrow[0,1]$ which satisfies the following properties $[29,30]$ :

- $\quad$ Commutativity: $\mathrm{T}(a, b)=\mathrm{T}(b, a)$

- $\quad$ Monotonicity: $\mathrm{T}(a, b) \leq \mathrm{T}(c, d)$ if $a \leq c$ and $b \leq d$

- $\quad$ Associativity: $\mathrm{T}(a, \mathrm{~T}(b, c))=\mathrm{T}(\mathrm{T}(a, b), c)$

- $\quad$ The number 1 acts as identity element: $\mathrm{T}(a, 1)=a$

Since inference system is based on the evaluation of all rules, the rules can be combined. The fuzzy inference for the first rule is illustrated in Figure 6. The input for the defuzzification process is the aggregated output fuzzy set and the output is a single crisp number.

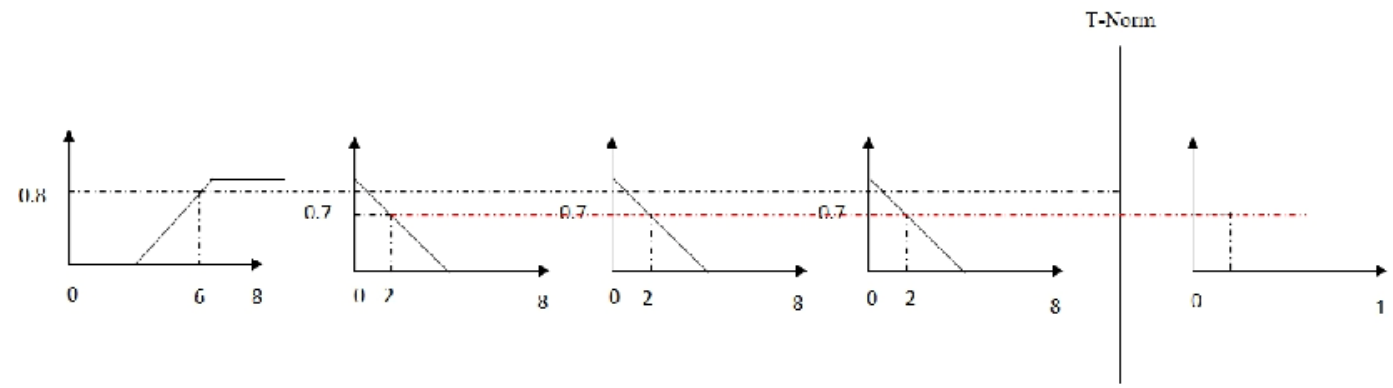

Fig 6: Mamdani fuzzy inference system for the first rule 
Figure 7 shows the defuzzification of rules in Matlab software. This figure shows that which rules must be considered and how each member functions impact to the output result.

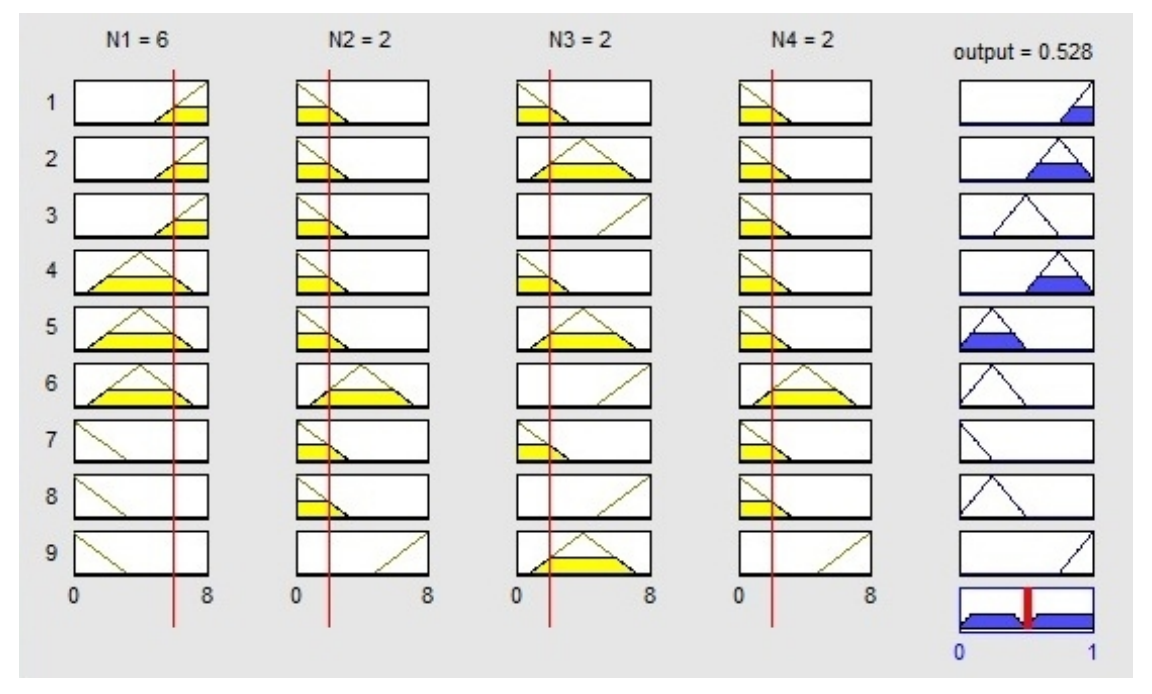

Fig 7: Fuzzy inference system using Matlab

Centroid method [31] is used to defuzzify the system output. The overall centroid of $\mathrm{N}$ overlapping areas $A_{i}$ for $\mathrm{i}=1,2 \ldots \mathrm{N}$ is given as equation Eq. (2).

$$
\alpha=\frac{\int_{Z} \mu_{A}(x) z d z}{\int_{Z} \mu_{A}(x) d z}
$$

Where $A_{i}$ and $\overline{X_{i}}$ are the overlapped area and centroid from the triangles achieved in the $i^{\text {th }}$ rule, respectively. The centroid and area are calculated for each triangle. This process is repeated for other inference rules where the inputs are referred to obtain an area comprised of overlapped trapeziums. The defuzzification process generates a centroid value that represents the rank of being a node faulty.

\section{COMPARISON}

Many fault detection methods have been proposed. Each of these methods has the own advantages and disadvantages. Jeng-Yang Wuet al. [4] proposed a method to detect the faulty node according to majority voting that needs fusion center to detecting faulty nodes in each time interval. The deployed sensor network in these applications may need to report its decision at every time step [4].Considering what was mentioned above, this method cannot be useful in all environments. Our new approach does not have this shortcoming, as it does not need computation of every time step. KuiRenetal. [2]applied majority voting for faulty node detection in a secure event boundary detection scheme (SEBD).This scheme is in fact a scheme to random events boundary detection in a secure mode and uses majority rule to prevent the effect of faulty nodes. In this scheme within the specified period, the number of sensed " 1 " is divided by the total sensed values, which is rating variable method. If the result has significant difference from the rate variables of neighbouring nodes, this node is defined as faulty node. In this scheme, each node 
has a table to keep the sensed values of the neighbouring nodes. The energy consumption to complete this table in each node is calculated by the equation Eq. (3).

$$
\text { Energy consumption }=(n * \lambda)+\lambda
$$

Where $\mathrm{n}$ is the number of the sent bits. For example, if the time step is eight, $\mathrm{n}-8$ then $\lambda$ is energy consumption per sending each bit. Energy consumption for the formation of the table in the proposed fuzzy fault detection method is calculated by equation Eq. (4).

$$
\text { Energy consumption_fuzzy }=(2 * \lambda)+\lambda=3 \lambda
$$

Figure 8 shows the proposed fuzzy fault detection method has less energy consumption than the other methods.

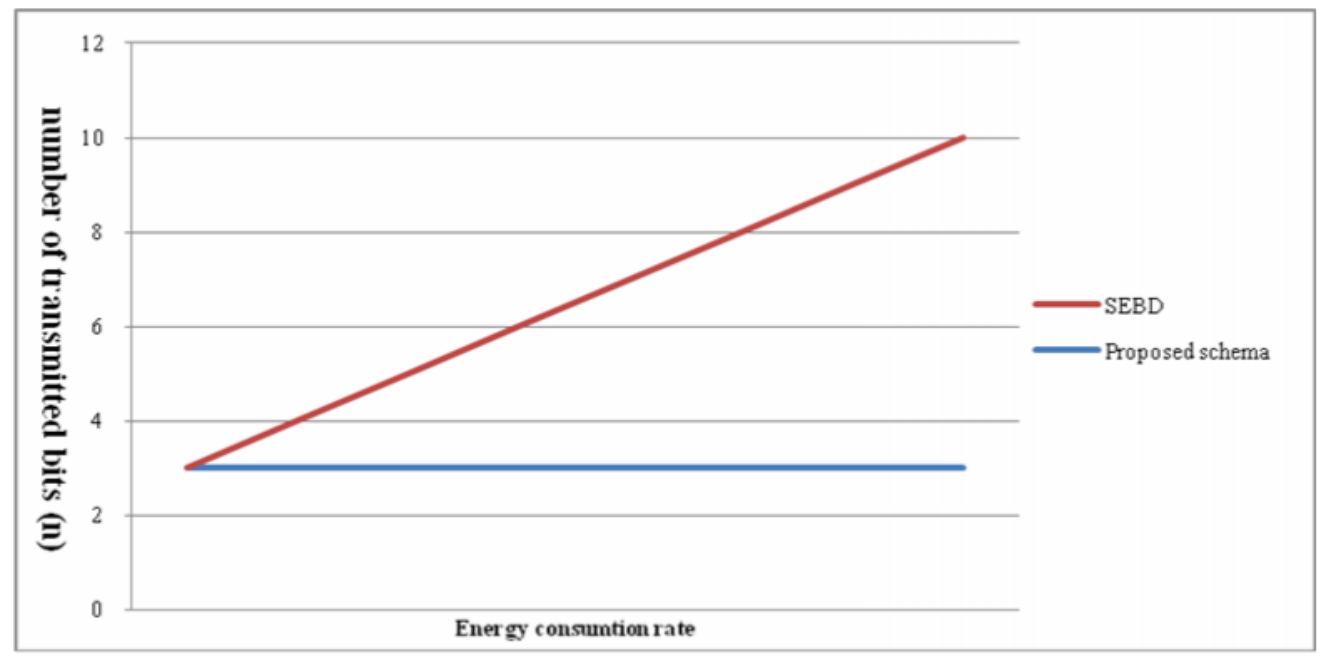

Fig 8: Comparing of energy consumption

In the proposed fuzzy fault detection method, the order of computational complexity is $\mathrm{O}$ (1). Since, the fuzzy inference engine is used in the proposed method, all rules compare with inputs are in parallel. Thus, the computational complexity is $\mathrm{O}$ (1). Computational complexity of the SEBD in terms of theory would not be less than $\mathrm{O}(\mathrm{n})$. Therefore, the computational complexity of the proposed fuzzy fault detection method is surprisingly improved, when compared with the other methods, as shown in Table 2. 
Table 2: Comparing proposed fuzzy fault detection method with the other methods

\begin{tabular}{|c|c|c|c|}
\hline $\begin{array}{l}\text { PROPOSED FUZZY } \\
\text { FAULT DETECTION } \\
\text { METHOD }\end{array}$ & SEBD [2] & $\begin{array}{c}\text { SCHEMA WITH USE } \\
\text { OF FUSION CENTRE } \\
{[4]}\end{array}$ & PARAMETERS \\
\hline Low & MEDIUM & RATHER HIGH & $\begin{array}{l}\text { COMPUTATIONAL } \\
\text { COMPLEXITY }\end{array}$ \\
\hline LOW & MEDIUM & RATHER HIGH & $\begin{array}{c}\text { ENERGY } \\
\text { CONSUMPTION }\end{array}$ \\
\hline $\mathrm{HIGH}$ & MEDIUM & MEDIUM & PRECISION \\
\hline
\end{tabular}

\section{CONCLUSION AND FUTURE WORK}

The proposed fault detection method uses the majority voting technique based on fuzzy logic to detect faulty nodes. In majority voting method, if a node sensed value has a significant difference with its neighbour's values in a specific period; it would be detected as a faulty node. Therefore, using the good characteristics of fuzzy logic can improve the accuracy of detecting faulty nodes in WSN. The proposed fuzzy fault detection method can increase the precision in detecting faulty nodes and it decrease computational complexity of calculation, end-to-end delay, and energy consumption, which resulted in network life increasing.

In the real environment, sensor nodes could be compromised and controlled by the attacker [32]. In these types of environment, malicious compromised nodes can exist. The effects of this type of nodes can be caused unexpected network attacks. Under this attack, a compromised node may try to impersonate another node at a different location, and it identifies itself as a neighbour node for voting. The semantic technology can be used to overcome this type of attacks. Ontology is the major element of semantic technology and illustrates and makes clear a vocabulary of terms. With the aid of ontology sensor nodes can be annotated to specify the neighbour relation between nodes. A sample of annotation is as follows.

$$
\begin{aligned}
\text { Subject } \rightarrow \text { Predicate } & \rightarrow \text { Object: Node } \rightarrow \text { is one-hop neighbour } \rightarrow \text { Next Node: } \\
& n_{1} \rightarrow \text { Is one-hop neighbour } \rightarrow n_{2}
\end{aligned}
$$

In this approach the neighbour relation between nodes will be defined and it will be specify that $n_{1}$ is the neighbour of $n_{2}$. Therefore, voting can be used to detect if $n_{1}$ is a faulty node. 


\section{REFERENCES}

[1] I. F. Akyildiz and I. H. Kasimoglu, "Wireless sensor and actor networks: Research challenges," Ad Hoc Networks J. (Elsevier), vol. 2, no. 4, pp.351-367, Oct. 2004.

[2] Namarta Kapoor, Nitin Bhatia, Sangeet Kumar, Simranjeet Kaur, "Wireless Sensor Networks: A Profound Technology," IJCST Vol. 2, Iss ue 2, pp.211-215, June 2011.

[3] A. Sharma, R. Chaki, U. Bhattacharya, "Applications of wireless sensor network in Intelligent Traffic System: A review," IEEE 3rd International Conference on Electronics Computer Technology (ICECT), pp. 53 - 57, April 2011.

[4] S.J., Isaac, G.P. Hancke, H. Madhoo, A. Khatri, "A survey of wireless sensor network applications from a power utility's distribution perspective," In AFRICON, pp. 1-5, 2011.

[5] K. Ren, K. Zeng, and W. Lou, "Secure and Fault-Tolerant Event Boundary Detection in Wireless Sensor Networks", IEEE TRANSACTIONS ON WIRELESS COMMUNICATIONS, VOL. 7, NO. 1, pp. 354 - 363, JANUARY 2008.

[6] J. Chen, Sh. Kher, and A. Somani, "Distributed Fault Detection of Wireless Sensor Networks," Proc Workshop DIWANS, Los Angeles, USA: ACM Pres, pp. 65-72, 2006.

[7] J-Y. Wu, D-R Duh, T-Y Wang and L-Y Chang, " On-Line Sensor Fault Detection Based on Majority Voting in wireless sensor networks," in Proc. the 24th Workshop on Combinatorial Mathematics and Computation Theory, National Chi Nan University, Puli, Nantou, Taiwan, pp. 292-297, April, 2007.

[8] Q. Ma, X. Zhao, Y. Liu, "Self-diagnosis for large scale wireless sensor networks," In INFOCOM, IEEE Proceedings, pp. 1539 - 1547, April 2011.

[9] I. Gupta, D. Riordan, S. Sampalli, "Cluster-head election using fuzzy logic for wireless sensor networks," in: Proceedings of the 3rd Annual Communication Networks and Services Research Conference, IEEE Computer Society, Washington, DC, USA, 2005, pp. 255-260.

[10] J. Kim, S. Park, Y. Han, T. Chung, "CHEF: cluster head election mechanism using fuzzy logic in wireless sensor networks," ICACT pp. 654-659, 2008.

[11] H.Y. Lee, T.H. Cho, "Fuzzy logic based key disseminating in ubiquitous sensor networks," in: 10th International Conference on Advanced Communication Technology (ICACT), Institute of Electrical and Electronics Engineers (IEEE), USA, pp. 958-962, 2008.

[12] B. Kim, H. Lee, T. Cho, "Fuzzy key dissemination limiting method for the dynamic filtering-based sensor networks," ICIC, pp. 263-272, 2007.

[13] B. Lazzerini, F. Marcelloni, M. Vecchio, S. Croce, E. Monaldi, "A fuzzy approach to data aggregation to reduce power consumption in wireless sensor networks," in: Fuzzy Information Processing Society, 2006, NAFIPS 2006, Annual Meeting of the North American, Institute of Electrical and Electronics Engineers (IEEE), USA, pp. 436-441, 2006.

[14] J.M. Kim, T.H. Cho, "Routing path generation for reliable transmission in sensor networks using ga with fuzzy logic based fitness function," in: Proceedings of the 2007 International Conference on Computational Science and Its Applications - Volume Part III, Springer-Verlag, Berlin, Heidelberg, pp. 637-648, 2007.

[15] S.-Y. Chiang, J.-L. Wang, "Routing analysis using fuzzy logic systems in wireless sensor networks," KES 966-973, 2008.

[16] Q. Ren, Q. Liang, "Fuzzy logic-optimized secure media access control (FSMAC) protocol wireless sensor networks," in: Proceedings of the 2005 IEEE International Conference on Computational Intelligence for Homeland Security and Personal Safety (CIHSPS), Institute of Electrical and Electronics Engineers (IEEE), USA, pp. 37-43, 2005.

[17] S.A. Munir, Y.W. Bin, R. Biao, M. Jian, "Fuzzy logic based congestion estimation for QoS in wireless sensor network," in: Wireless Communications and Networking Conference (WCNC), Institute of Electrical and Electronics Engineers (IEEE), USA, pp. 4336-4341, 2007.

[18] F. Xia, W. Zhao, Y. Sun, Y.-C. Tian, "Fuzzy logic control based QoS management in wireless sensor/actuator networks", Sensors 7 (12), pp. 3179-3191, 2007.

[19] L. Lam and S. Suen, "Application of majority voting to pattern recognition: an analysis of its behavior and performance," Systems, Man and Cybernetics, Part A: Systems and Humans, IEEE Transactions on, vol. 27, no. 5, pp. 553-568, Sep 1997.

[20] M. Xie, S. Han, B. Tian, S. Parvin," Anomaly detection in wireless sensor networks: A survey," Journal of Network and Computer Applications Volume 34, Issue 4, Pages 1302-1325, July 2011.

[21] L.A. Klein, Sensor and Data Fusion Concepts and Applications. SPIE, Apr. 1993. 
International Journal Of Advanced Smart Sensor Network Systems ( IJASSN ), Vol 2, No.4, October 2012

[22] M. Ding, D. Chen, K. Xing, and X. Cheng, "Localized event detectionin sensor networks," in Proc. IEEE INFOCOM, Mar. 2005.

[23] P. Bonissone, “A Fuzzy Sets Based Linguistic Approach: Theory and Applications,” in Proceedings of the 12th Conference on Winter Simulation, California, pp. 275-284, 1980.

[24] T.J. Ross, "Fuzzy Logic With Engineering Applications," Third Edition, A John Wiley and Sons, Ltd., Publication, ISBN: 978-0-470-74376-8, 2010.

[25] L.A. Zadeh, "Fuzzy logic," Computing \& Processing (Hardware/Software), IEEE, Volume: 21 Issue: 4, pp. $83-93,1988$.

[26] Zh.J. Bose, "BK: Evaluation of membership functions for fuzzy logic controlled induction motor drive,” In: IECON Proceedings (Industrial Electronics Conference), vol. 1, pp. 229-234, 2002.

[27] W. Pedrycz, "Why triangular membership functions?," Fuzzy sets and Systems, Elsevier, Volume 64, Issue 1, pp. 21-30, 1994.

[28] M. Negnevitsky, Artificial intelligence: A guide to intelligent systems, Addison-Wesley, Reading, MA, 2001.

[29] P. Hájek, "Towards metamathematics of weak arithmetics over fuzzy logic," Oxford Journals, Mathematics \& Physical Sciences, Logic Journal of the IGPL, Volume 19, Issue 3, pp. 467-475, 2010.

[30] À. Garcı'a-Cerdaña, E. Armengol , F. Esteva, "Fuzzy Description Logics and t-norm based fuzzy logics," International Journal of Approximate Reasoning Volume 51, Issue 6, Pp. 632-655, Elsevier, July 2010.

[31] B. Kosko, "Neural Networks and F u q Systems: A Dynamical Systems Approach to Machine Intelligence," Prentice Hall, New Jersey, 1992.

[32] K. Ren, K. Zeng, and W. Lou, "A new approach for random key pre-distribution in large-scale wireless sensor networks," J. WirelessCommun.and Mobile Computing (special issue on wireless networks security), vol. 6, no. 3, pp. 307-318, 2006.

\section{Authors}

Saeed Javanmardi was born in Shiraz, Iran. He received the MSC in computer engineering from Dezful University, Iran. His research interests are in Semantic Web, Fuzzy theory, Grid resource discovery based on P2P, Service oriented architecture and WSN faulty node detection.

Ali Barati is currently a member of Department of Computer Engineering, Dezful Branch, Islamic Azad University, Dezful, Iran. He is currently working toward the Ph.D. degree in Computer Software Engineering at Qazvin Branch, Islamic Azad University, Qazvin, Iran. His research interests include AD-HOC networks, wireless sensor networks, high speed networks and fault tolerant systems.

Seyyed Jalaleddin Dastgheib was born in Shiraz, Iran, in 1986. He received the B.Sc. degree in Computer Software Engineering from Tabriz University, Tabriz, Iran, in 2008. He also received the M.Sc. degree in Computer Hardware Engineering from Dezful Branch, Islamic Azad University, Dezful, Iran, in 2011. His research interests include computer arithmetic, wireless sensor network, fuzzy systems, and fault tolerant systems.

Iman Attarzadeh received his M.Sc. in Computer Architecture from Islamic Azad University, Tehran, Iran, in 2005. he was the Head of Department of Computer Science at the University in 2005. His current research interests include software engineering (project management, formal method, algorithm design, and embedded systems), soft computing theories and techniques, image processing, and computer Architecture.

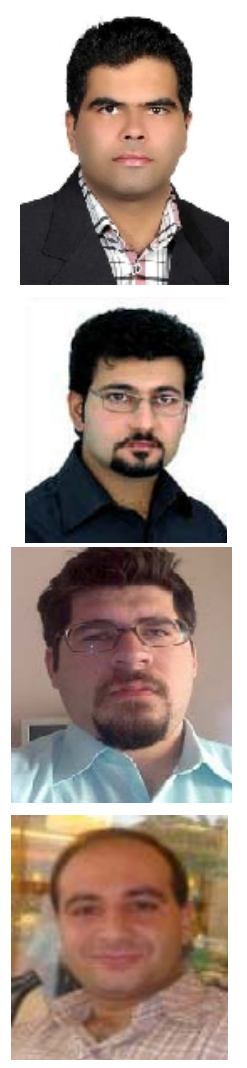

